\title{
Economic evaluation of the primary recovery of tetracycline with traditional and novel aqueous two-phase systems
}

\author{
Mario A. Torres-Acosta ${ }^{\mathrm{a}, \mathrm{b}}$, Jorge F.B. Pereira ${ }^{\mathrm{c}}$, Mara G. Freire ${ }^{\mathrm{d}}$, José M. Aguilar-Yáñez ${ }^{\mathrm{b}}$, \\ João A.P. Coutinho ${ }^{\mathrm{d}}$, Nigel J. Titchener-Hooker ${ }^{\mathrm{a}}$, Marco Rito-Palomares, ${ }^{\mathrm{e}}$, \\ a The Advanced Centre for Biochemical Engineering, Department of Biochemical Engineering, University College London, Torrington Place, London WC1E 7JE, UK \\ ${ }^{\mathrm{b}}$ Tecnológico de Monterrey, Escuela de Ingeniería y Ciencias, Ave. Eugenio Garza Sada 2501 Sur, Monterrey, NL 64849, Mexico \\ ${ }^{\mathrm{c}}$ Department of Bioprocess and Biotechnology, School of Pharmaceutical Sciencies, Universidade Estadual Paulista, Rodovia Araraquara-Jau Km. 01, CEP 14801-902, \\ Araraquara, SP, Brazil \\ ${ }^{\mathrm{d}}$ CICECO - Aveiro Institute of Materials, Chemistry Department, University of Aveiro, Campus Universitário de Santiago, 3810-193 Aveiro, Portugal \\ e Tecnológico de Monterrey, Escuela de Medicina y Ciencias de la Salud, Av. Morones Prieto 3000 Pte, Col. Los Doctores, Monterrey, NL 64710, Mexico
}

\section{A R T I C L E I N F O}

\section{Keywords:}

Tetracycline

Aqueous two-phase system (ATPS)

Ionic liquids

Economic analysis

Monte Carlo simulations

\begin{abstract}
A B S T R A C T
Antibiotics are a key pharmaceutical to inhibit growth or kill microorganisms. They represent a profitable market and, in particular, tetracycline has been listed as an essential medicine by the WHO. Therefore it is important to improve their production processes. Recently novel and traditional aqueous two-phase systems for the extraction have been developed with positive results. The present work performs an economic analysis of the production and recovery of tetracycline through the use of several ATPS through bioprocess modeling using specialized software (BioSolve, Biopharm Services Ltd, UK) to determine production costs per gram (CoG/g). First, a virtual model was constructed using published data on the recovery of tetracycline and extended to incorporate uncertainties. To determine how the model behaved, a sensitivity analysis and Monte Carlo simulations were performed. Results showed that ATPS formed by cholinium chloride $/ \mathrm{K}_{3} \mathrm{PO}_{4}$ was the best option to recover tetracycline, as it had the lowest CoG/g (US\$ 672.83/g), offered the highest recovery yield (92.42\%), second best sample input capacity (45\% of the ATPS composition) and one of the lowest materials contribution to cost. The ionic liquid-based method of ATPS is a promising alternative for recovering tetracycline from fermentation broth.
\end{abstract}

\section{Introduction}

Antibiotics are widely used to inhibit growth or kill infecting microorganisms in humans and animals. They represent a globally profitable market; in 2009 sales generated US\$ 42 billion [1]. There are multiple types of antibiotics, for example $\beta$-lactams, cephalosporins, aminoglycosides, macrolides and tetracyclines (TC's) [2]. Tetracyclines are bacteriostatic antimicrobials produced mainly through fermentation by the Streptomyces genre $[3,4]$ and are considered broad spectrum antibiotics. They act by binding to ribosome regions and inhibit protein synthesis, preventing the growth of bacteria and, ultimately, killing them [5]. Tetracycline is listed as an essential medicine by the World Health Organization [6], which makes identifying effective and economic routes for its production a priority.

Several methods for the recovery of Tetracycline (TC) from the fermentation broth of Streptomyces aureofaciens have been proposed. Most of them rely on recovery by crystallization of the antibiotic $[7,8]$, chromatographic techniques or liquid-liquid extraction with organic solvents [9]. The latter may be harmful to humans. An alternative is the use of aqueous two-phase systems (ATPS), which have been used previously for the recovery of a wide variety of biomolecules $[10,11]$, for example antibiotics [12-14], metal ions [15], antibodies [16], organic compounds [17], proteins [18,19], cell debris (González- [20] and stem cells [21].

Amongst ATPS, there are different classes depending on the phaseforming components required to form two liquid immiscible phases in aqueous media, each with different strengths and weaknesses [22]. Traditional systems comprise of a polymer and a salt or two different polymers. More recent systems have been reported with ionic liquid (IL)/salt, IL/polymer or IL/carbohydrate combinations [23], or with low molecular weight alcohol/salt mixtures $[24,10]$. Recently, a study was published in which the recovery capabilities of traditional (Polymer/Salt) and novel (IL/Salt) ATPS for TC were evaluated [14]. The novel techniques (ATPS-based) have the potential to reduce costs in

\footnotetext{
* Corresponding author.

E-mail address: mrito@itesm.mx (M. Rito-Palomares).
} 


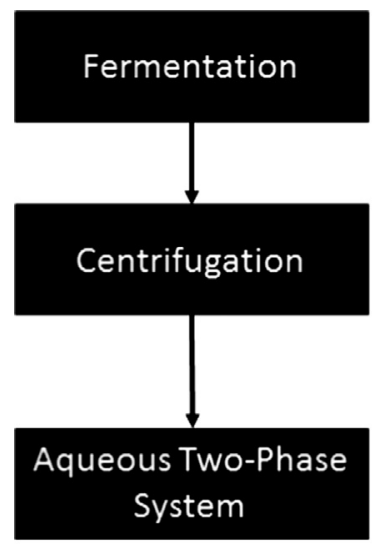

Streptomyces aureofaciens

fermentation (68.19 L)

Medium E. Time: 4.5 days.

Titer: $1.2 \pm 0.1 \mathrm{~g} / \mathrm{L}$

Recovery: $85 \%$

Time: $8 \mathrm{~h}$

ATPS 1

- PEG: $54.91 \%$

- $\quad[\mathrm{Ch}] \mathrm{Cl}: 24.95 \%$

- Sample: $10 \%$

- Water: $\mathbf{1 0 . 1 4} \%$

\section{ATPS 2}

- PEG: $49.88 \%$

- [Ch]Bic: $34.93 \%$

- Sample: $15 \%$

- Water: $0.19 \%$
Recovery: $81.63 \% \pm 5.26$

KTC: $0.21 \pm 0.03$

Volume to recover: $\mathbf{5 5 \%}$

Recovery: $82.54 \% \pm 11.03$

KTC: $3.98 \pm 0.41$

Volume to recover: $55 \%$
ATPS 3

- PEG: $40.03 \%$

- $\mathrm{Na}_{2} \mathrm{SO}_{4}: 7.48 \%$

Sample: $\mathbf{5 2 . 5} \%$

Water: $0 \%$

ATPS 4

- [Ch]Cl: $20.04 \%$

- $\mathrm{K}_{3} \mathrm{PO}_{4}: 35.02 \%$

Sample: $45 \%$

Water: $0 \%$
Recovery: $54.67 \% \pm 6.55$

KTC: $17.78 \pm 1.25$

Volume to recover: $81 \%$

Recovery: $92.42 \% \pm 2.84$

KTC: $45.95 \pm 1.66$

Volume to recover: $42 \%$

Fig. 1. Process diagram for the primary recovery of Tetracycline based on ATPS. Critical parameters for model construction for each unit operation are shown. KTC: Partition coefficient for tetracycline.

Table 1

Materials used in the construction of the Tetracycline production model (fermentation medium and aqueous two-phase systems). Concentration for ATPS construction is presented in Fig. 1.

\begin{tabular}{|c|c|c|c|}
\hline \multicolumn{4}{|l|}{ Medium E } \\
\hline Material & Concentration (g/L) & $\begin{array}{l}\text { Commercial } \\
\text { Presentation }(\mathrm{kg})\end{array}$ & $\begin{array}{l}\text { Cost (US\$ } \\
\text { dollars) }\end{array}$ \\
\hline Sucrose & 40 & 5 & 81.10 \\
\hline $\mathrm{C}_{6} \mathrm{H}_{5} \mathrm{O}_{7} \cdot \mathrm{H} 2 \mathrm{O}$ & 12.8 & 5 & 112.00 \\
\hline$\left(\mathrm{NH}_{4}\right)_{2} \mathrm{SO}_{4}$ & 6 & 1 & 44.00 \\
\hline $\mathrm{MgSO}_{4} \cdot 7 \mathrm{H}_{2} \mathrm{O}$ & 0.25 & 5 & 116.00 \\
\hline $\mathrm{KH}_{2} \mathrm{PO}_{4}$ & 0.15 & 2.5 & 108.10 \\
\hline $\mathrm{CaCO}_{3}$ & 11 & 2.5 & 191.50 \\
\hline $\mathrm{MnSO}_{4} \cdot 4 \mathrm{H}_{2} \mathrm{O}$ & 0.01 & 50 & 1138.27 \\
\hline $\mathrm{ZnSO}_{4} \cdot 7 \mathrm{H}_{2} \mathrm{O}$ & 0.04 & 2.5 & 84.50 \\
\hline $\mathrm{K}_{2} \mathrm{Cr}_{2} \mathrm{O}_{7}$ & $0.016(\mathrm{mg} / \mathrm{L})$ & 2 & 72.60 \\
\hline \multicolumn{4}{|l|}{ ATPS } \\
\hline Material & Concentration $(\% w / w)$ & $\begin{array}{l}\text { Commercial } \\
\text { Presentation }(\mathrm{kg})\end{array}$ & $\begin{array}{l}\text { Cost (US\$ } \\
\text { dollars) }\end{array}$ \\
\hline $\begin{array}{l}\text { Polyethylene } \\
\text { glycol } 600\end{array}$ & $\begin{array}{l}\text { Concentration depends } \\
\text { on the ATPS being }\end{array}$ & 20 & 385.00 \\
\hline $\begin{array}{l}\text { Cholinium } \\
\text { chloride }\end{array}$ & prepared & 1000 & 1000.00 \\
\hline $\begin{array}{l}\text { Cholinium } \\
\text { bicarbonate }\end{array}$ & & 15 & 441.59 \\
\hline $\mathrm{Na}_{2} \mathrm{SO}_{4}$ & & 12 & 142.04 \\
\hline $\mathrm{K}_{3} \mathrm{PO}_{4}$ & & 10 & 248.50 \\
\hline
\end{tabular}

Table 2

Scenarios used for the sensitivity analysis.

\begin{tabular}{llll}
\hline Variable & \multicolumn{2}{l}{ Scenarios } & \\
\cline { 2 - 4 } & \multicolumn{1}{l}{ Best } & Base & Worst \\
\hline Titer (g/L) & 1.1 & 1.2 & 1.3 \\
DSP Yield (\%) & Depends on the ATPS analyzed: & \\
ATPS 1 & 86.89 & 81.63 & 76.37 \\
ATPS 2 & 93.57 & 82.54 & 71.51 \\
ATPS 3 & 61.22 & 54.67 & 48.12 \\
ATPS 4 & 95.26 & 92.42 & 89.58 \\
Material Cost variation $( \pm \%)$ & -25 & 0 & +25 \\
\hline
\end{tabular}

the recovery of the antibiotic, as demonstrated before with other biomolecules through the use of bioprocess modeling $[25,26]$. In addition, previous works which employed cholinium-based IL have demonstrated low toxicity and ecological impact, and also the ability to remove the largest contaminant (Chlortetracycline) from the fermentation broth [29,30-32].

Bioprocess modeling provides a powerful computational tool that allows the researcher to create virtual models, based on experimental or reported data to emulate real bioprocesses. The models can be integrated in order to determine production costs and to understand how these respond when critical parameters are modified, ultimately reducing production costs. This strategy has been used successfully to determine the impact of the use of stainless steel versus single-use equipment [33], to determine the best strategy to harvest a perfusion reactor $[34,35]$, and to evaluate the impact of optimizing certain production parameters [26], the use of different purification strategies [27] or to optimize the production of monoclonal antibodies [36,37].

There are several commercial software packages that perform this type of analyses, each with its own advantages and disadvantages [38]. The present work utilizes BioSolve Process (BioPharm Services Ltd, Chesham, Buckinghamshire, UK), which is an Excel-based software package that is heavily focused on biotechnological applications and is able to perform economic analyses using data fed by the user, but it also includes updated costs for equipment and materials from different suppliers. The latter was selected for this study as it can perform bioprocess modeling and economic analyses oriented towards biotechnological products.

This study uses the experimental data, obtained during the evaluation of traditional versus novel ionic-liquid-based ATPS for the recovery of TC [14], to perform an economic analysis designed to elucidate how the production costs per gram $(\mathrm{CoG} / \mathrm{g})$ responds to key variables and parameters and to determine if the use of a novel form of ATPS based on an ionic liquid leads to a lower cost than traditional ATPS. For this purpose, a base model was created using BioSolve Process, to which variations in parameters were made through a sensitivity (local robustness) and Monte Carlo (global robustness) analysis in order to understand the behavior of the model. A series of comparisons were then made in order to determine how each ATPS variant behaves and which system offers the best option for tetracycline recovery. Moreover, as each ATPS had different capabilities, the impact of having small or large variations for a certain parameter was evaluated.

\section{Materials and methods}

\subsection{Model Set-Up and deterministic analysis}

This study focused on contrasting the CoG/g incurred when using traditional and novel ATPS approaches for the primary recovery of TC, 
a)

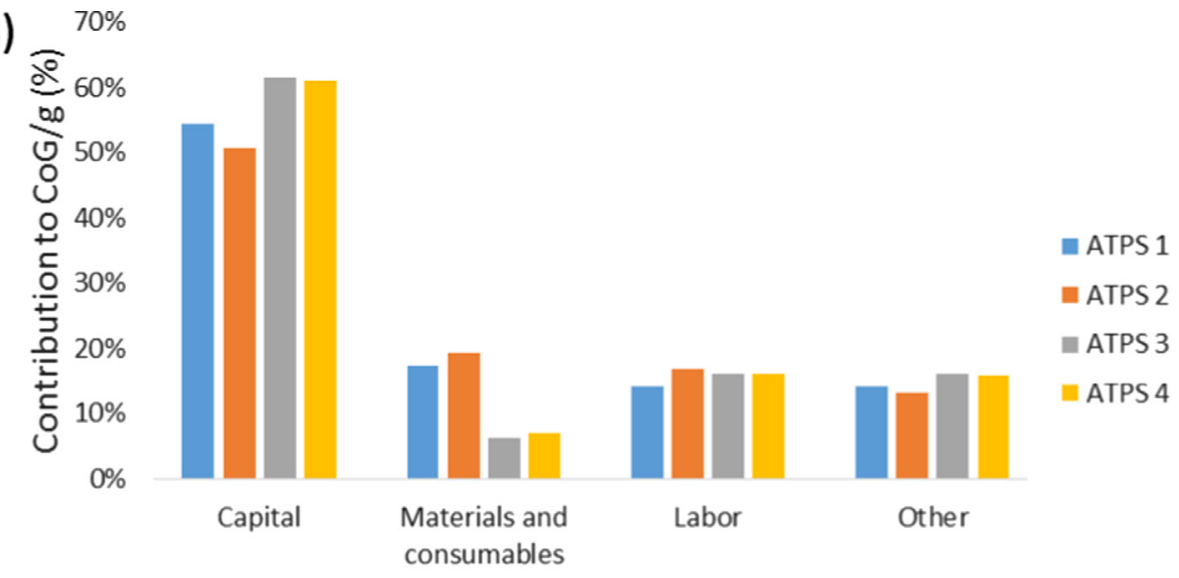

Cost Categories

b)

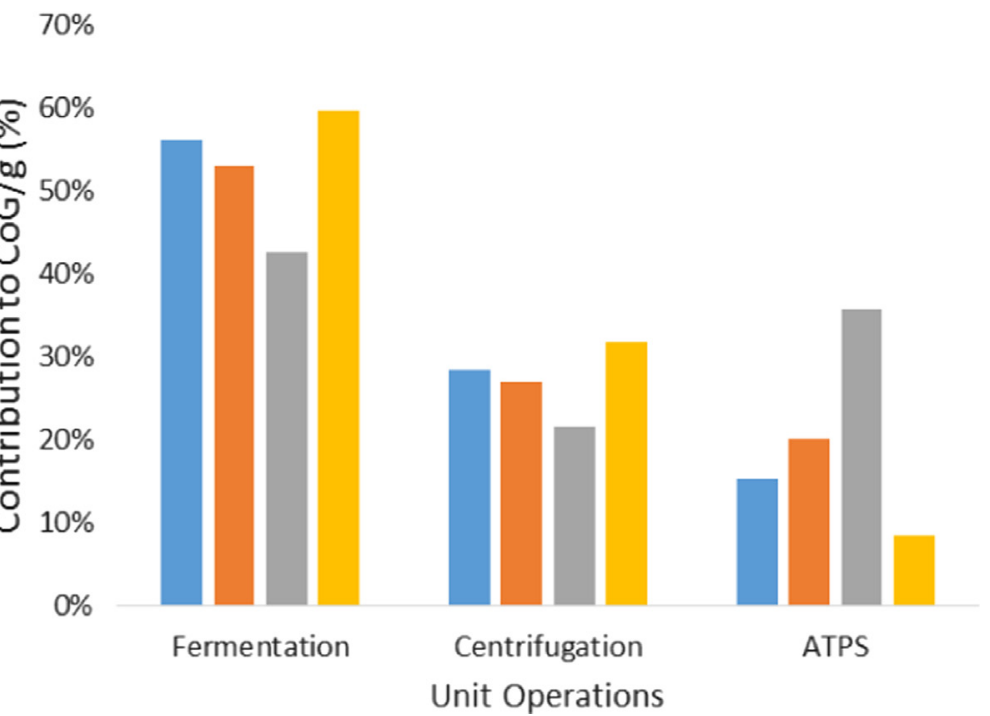

ATPS 1

ATPS 2

ATPS 3

ATPS 4

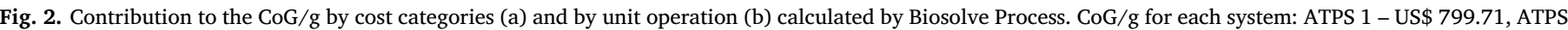
2 - US\$ 847.09, ATPS 3 - US\$ 1053.38, ATPS 4 - US\$ 627.83. CoG/g: Cost of Goods per gram.

ATPS compositions and partition results were obtained from literature [14]. Models were constructed using BioSolve Process (Biopharm Services Ltd, Chesham, Buckingham, UK). For the bioprocess models (Fig. 1) only the fermentation and primary recovery were created and comprised of a seed reactor to grow an inoculum and a fermentation reactor where Streptomyces aureofaciens secreted the tetracycline product. For biomass removal a centrifuge (Biosolve default was selected as the objective was to evaluate ATPS capabilities) was used and the supernatant was loaded to one of four alternative ATPS (Fig. 1, namely ATPS 1: Polyethylene glycol 600 (PEG600)/Cholinium Chloride ([Ch]Cl); ATPS 2: PEG600/Cholinium Bicarbonate ([Ch]Bic); ATPS 3: PEG600/ $\mathrm{Na}_{2} \mathrm{SO}_{4}$; ATPS 4: $[\mathrm{CH}] \mathrm{Cl} / \mathrm{K}_{3} \mathrm{PO}_{4}$. There were 4 bioprocess models in total (1 per ATPS), which each had the same initial production bioreactor, but a different target output of tetracycline (TC) after the ATPS (as each ATPS had a different recovery yield). Operation and process parameters were taken for the fermentation [3] and recovery [14] from the literature. Technical characteristics of the equipment used are included in Supplementary Material 2 (sheet "Equipment").

Labor was set in the range of $10-15 \%$ of the total CoG/g [39] and the standard set of quality control tests from BioSolve Process were assumed ( $\mathrm{pH}$ determination, purity by SDS-PAGE and size-exclusion chromatography, residual nucleic acids by ELISA and turbidity). The equipment used by the modeled bioprocess was selected from Biosolve Process database, while the materials (medium E for fermentation and
ATPS components) were added and are presented in Table 1. For ATPS operation, stainless-steel tanks from Biosolve were considered. After the completion of the models a deterministic analysis was performed by obtaining the initial CoG/g and its breakdown for each of the four bioprocesses considered directly from Biosolve.

\subsection{Sensitivity analysis}

Several bioprocess parameters can impact the $\mathrm{CoG} / \mathrm{g}$ and it is critical to identify the most sensitive in order to develop strategies to maintain control and to achieve a stable CoG/g. The most often studied parameters are the titer, downstream processing yield (DSP yield), material costs, operator wages, sale price and target output $[34,35,40]$. Titer, DSP yield and materials cost variations were considered in this study.

To determine the robustness of the bioprocess models to variations in individual parameters, three scenarios (Table 2 were created; one for each of the parameters analyzed: titer $(\mathrm{g} / \mathrm{L})$, DSP yield $(\%)$ and materials costs variations $( \pm \%)$. The ranges for titer and DSP yield were created by taking the mean (base scenario) plus and minus one standard deviation (worst or base scenario, depending on the parameter being analyzed) for each parameter using the values reported in their respective publications $[3,14]$. For materials costs it has been reported that a variation of $\pm 25 \%$ can occur [35]. Each parameter was varied individually, in their respective Biosolve Process space, and the 
a)

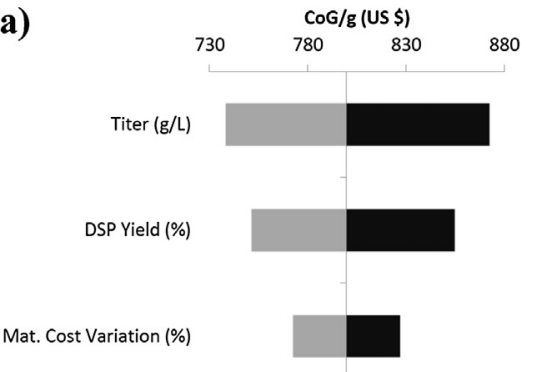

c)

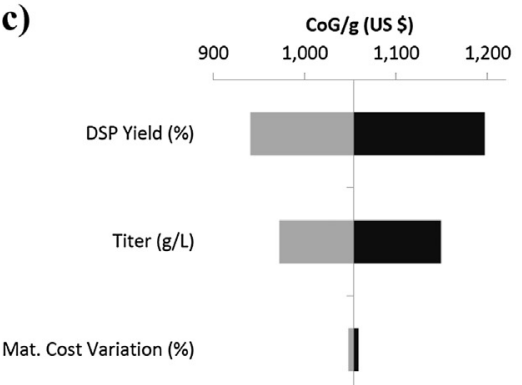

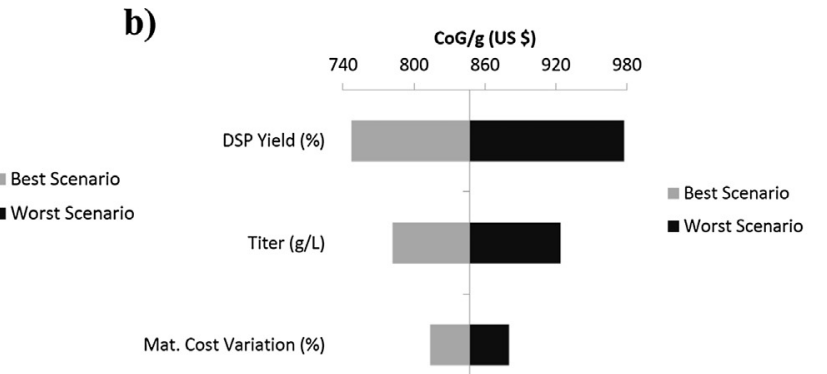

d)

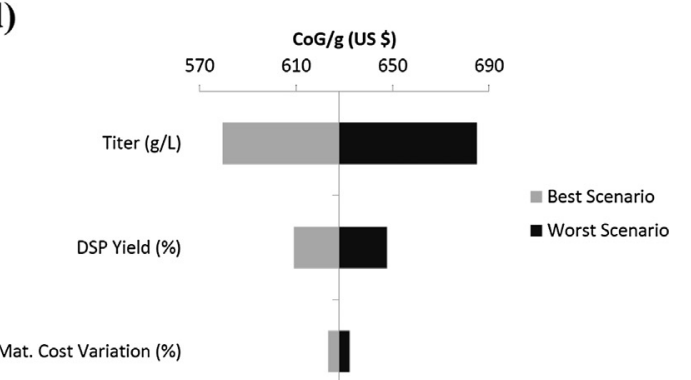

Fig. 3. Tornado plots. Results for the sensitivity analysis are summarized here. (a) ATPS 1, (b) ATPS 2, (c) ATPS 3 and (d) ATPS 4. DSP: Downstream processing; CoG/g: Cost of Goods per gram.

calculated CoG/g was registered for later analysis.

\subsection{Monte Carlo analysis}

After the identification of the critical parameters in the previous analysis, and in order to analyze the behavior of the models under the influence of uncertainty, the two most important parameters from the previous step were selected for a Monte Carlo analysis. This is a widely accepted strategy used to incorporate uncertainties into a bioprocess and to analyze their impact on economics [41]. To perform this analysis a program was written in Visual Basic (Microsoft Office 365 Pro Plus, Microsoft Corporation), included as supplementary material 1, that generated random values for each parameter under a triangular distribution $[42,43,40]$, while using as its limits the scenarios presented for the sensitivity analysis (Table 2. 300 simulation runs were used to generate a stable moving average, this limit has been tested before for models created in Biosolve Process and have resulted in not significant variations for outputs calculated (data not shown). ANOVA tests and ttests for mean differences were performed after the completion of the simulation runs to determine if the distributions for the CoG/g showed a statistically significant difference between all the ATPS. Linear models were constructed using $\mathrm{R}$ (where the independent variables were the values for each of the parameter analyzed and the response variable was de $\mathrm{CoG} / \mathrm{g}$ registered after each simulation) in order to summarize the complete set of data (one per ATPS) and to create a simpler form to understand the general behavior of the results obtained after the simulation runs.

\section{Results and discussion}

\subsection{Deterministic analysis}

The model constructed in Biosolve Process is presented as supplementary material 2. From this model, four base $\mathrm{CoG} / \mathrm{g}$ and their breakdowns per cost category and unit operation were obtained (Fig. 2), one for each ATPS analyzed. ATPS 4 had the lowest CoG/g and the 2nd smallest materials contribution, making it the most attractive system to use. Moreover, it also had the second largest sample input (ratio between sample and system weight) (Fig. 1. ATPS 2 had the 2nd highest cost (CoG/g) and the largest materials contribution, as it had one of the smallest sample input values (small sample per unit of material required). ATPS 3 provided an interesting result, as it had the highest cost, the largest sample input and the lowest materials contribution. Its high $\mathrm{CoG} / \mathrm{g}$ is explained by the low DSP yield which means that the cost is distributed across a small inventory of tetracycline, causing the $\mathrm{CoG} / \mathrm{g}$ to increase.

Fermentation is the unit operation that contributes the most to the cost (Fig. 2b), as it is expected as the equipment required is the most complex in the bioprocess. The second unit operation in contribution to the CoG/g is dependent on the ATPS being analyzed. ATPS 3 provided an expensive system providing a larger contribution that centrifugation, for ATPS 1, 2 and 4, this is the opposite. This reinforce the results that ATPS 3 has the lowest recovery of Tetracycline

\subsection{Sensitivity analysis}

The results for each of the ATPS analyzed were obtained and are presented in Tornado plots in Fig. 3. The top two parameters were titer and DSP yield in all cases, but it can be seen that for ATPS 1 and 4 the most critical parameter was titer, while for ATPS 2 and 3 it was the DSP yield.

From an economic perspective, the ATPS 4 was the most viable option (lowest CoG/g) for several reasons; it is a small system compared to the rest due to the proportion of sample input, it has the highest DSP yield with the lowest standard deviation, also ATPS 4 has the second lowest materials contribution to the CoG/g. The opposite for ATPS 3, it has the highest sample input but the lowest DSP yield, which was the most important parameter, as it dominates the contributions to the CoG/g. ATPS 1 and 2 were located between the outcomes of the other two systems, with a medium DSP yield. ATPS 2 had the highest standard deviation, which made it more sensitive to variations in the DSP yield. By contrast, ATPS 1 displayed a smaller variation range and was more sensitive to variations in titer.

\subsection{Monte Carlo analysis}

The distributions of the simulation runs results generated after performing simultaneous variations in the titer and DSP yield are 
a)

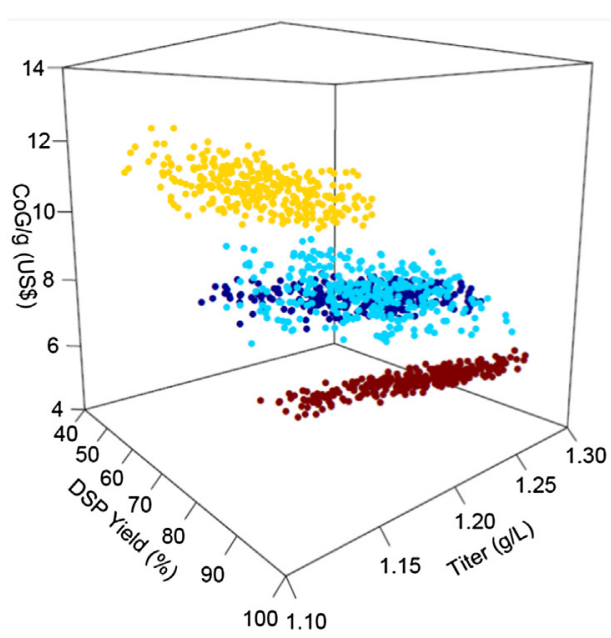

b)

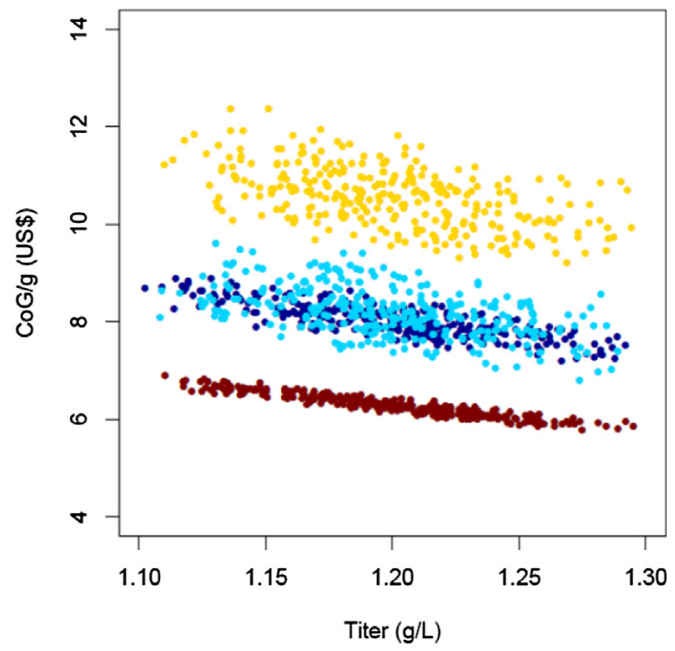

Fig. 4. Monte Carlo analysis results for ATPS 1 ( ), ATPS 2 ( ), ATPS $3(0)$ and ATPS 4 ( ), CoG/g is expressed in $\mathrm{x} 10^{2} \mathrm{USD} / \mathrm{g}$. a) Cost of Goods per gram (CoG/g) distribution as a function of titer and downstream processing (DSP) yield, b) CoG distribution as a function of titer and c) CoG distribution as a function of DSP yield.

c)

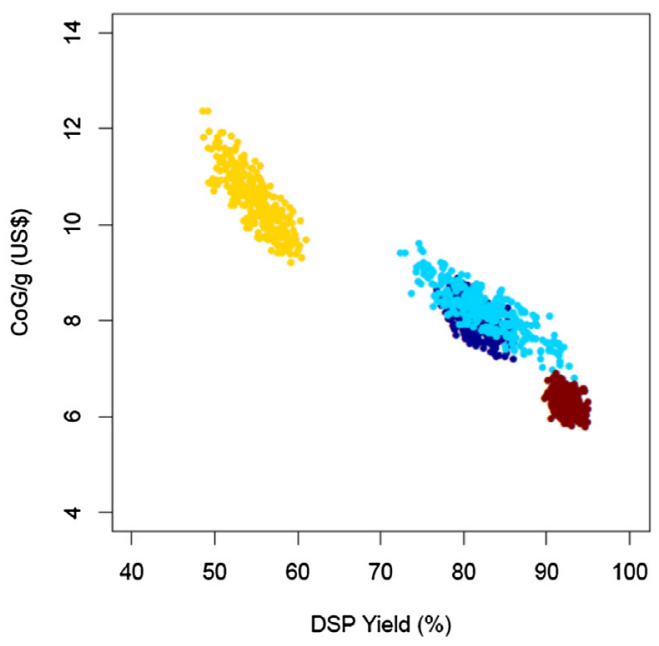

Table 3

Linear models for Monte Carlo analysis results. Linear regression were calculated for individual ATPS and for all the results simultaneously.

\begin{tabular}{llllll}
\hline & ATPS $^{*}$ & ATPS 2 $^{*}$ & ATPS 3 $^{*}$ & ATPS $^{*}$ & Complete $^{+}$ \\
\hline Parameter & Coefficient & Coefficient & Coefficient & Coefficient & Coefficient \\
Intercept & $2,406.70$ & $2,451.33$ & $3,164.59$ & $1,888.51$ & $2,489.36$ \\
Titer & -669.98 & -678.82 & -876.71 & -526.13 & -695.02 \\
Downstream & -9.81 & -9.89 & -19.31 & -6.80 & -10.65 \\
$\quad$ processing & & & & & \\
$\quad$ Yield & & & & &
\end{tabular}

All coefficients are statistically significant for $\alpha<0.01$.

* ATPS 1: PEG600/[Ch]Cl; ATPS 2: PEG600/[Ch]Bic; ATPS 3: PEG600/ Na2SO4; ATPS 4: [Ch]Cl/K3PO4.

+ Complete: linear regression of all the data simultaneously.

presented in Fig. 4 (a-c). Fig. 4a shows the complete tri-dimensional distribution, while Fig. $4 \mathbf{b}$ and $\mathbf{c}$ depict two-dimensional projections constructed in order to understand the individual contributions of the titer and DSP yield on the CoG/g. Four different distributions can be observed, each one corresponding to the individual ATPS analyzed. To understand fully the behavior of each distribution, linear models were constructed for each individual system analyzed and are summarized in Table 3.

The ANOVA and t-tests results revealed that all distributions of
CoG/g were statistically different to a critical value of $\alpha=0.01$, although linear models showed that ATPS 1 and 2 (Table 3 were very similar since their coefficients changed by less than a unit. This was confirmed by analyzing Fig. 4 where both distributions are seen to overlap.

After analysis of the linear models, it was clear that for every ATPS, the most significant parameter was the titer, followed by the DSP yield. This is logical as the four ATPS shared the same titer with a variation of $10 \%( \pm 0.1 \mathrm{~g} / \mathrm{L})$ providing a small range of variation with a large variation in production cost, therefore a larger coefficient for the linear models in Table 2. Contrary, DSP yield (for all the systems) had a variation of less than the $12 \%$ (each system with a different variation corresponding to their unique standard deviation), as it can be seen in Table 2, but also a small variation in $\mathrm{CoG} / \mathrm{g}$, providing a smaller coefficient in the linear models. For all the linear models, DSP yield coefficients behaved as expected, with ATPS 3 being the most affected, while it had the least impact on ATPS 4. From Fig. 4, it can be concluded that titer is more important than DSP yield as it can affect production cost while having a small absolute variation $( \pm 0.1 \mathrm{~g} / \mathrm{L})$. For a potential bioprocess set-up and with incorporation of ATPS, optimization of titer can represent an alternative to decrease production costs The evidence collected in this paper, it was confirmed that ATPS 4 is the most attractive of the four systems analyzed. In this particular model, the high DSP yield of ATPS 4 was achieved by solely using ionic 
liquids as phase-forming components. ATPS 1, 2 and 4 were constructed using cholinium-based ionic liquids ([Ch]Cl and [Ch]Bic). ATPS 4 achieved the lowest $\mathrm{CoG} / \mathrm{g}$, while the highest $\mathrm{CoG} / \mathrm{g}$ was obtained with a traditional ATPS composed of PEG600/ $\mathrm{Na}_{2} \mathrm{SO}_{4}$.

Clearly ionic-liquid-based ATPS provide an attractive alternative as they have a higher level of recovery than do traditional systems, while maintaining a low cost and system size. Ionic liquid-based ATPS have presented enhanced properties, namely the possibility to increase recovery yields and to improve selectivity [44]. Some of them also have a low ecological impact and toxicity. To this end, the chemical structures of the ionic liquids investigated play the most significant role. Ionic liquids derived through sustainable and easy procedures from natural sources can be seen as a good alternative when envisaging their use as phase-forming components to recover/purify value-added biomolecules. In particular, $[\mathrm{Ch}] \mathrm{Cl}$ has been categorized as "practically harmless" and [Ch]Bic as "harmless" [29]. They can have tunable hydrophobicity and charge density [45], making them a valuable tool for the future recovery of high value products. An additional advantage of an ATPS based on ionic liquids is the large sample loading capacity, which is above to that displayed by other reported systems [46,19], and it is above to the traditional ATPS analyzed here.

Furthermore, from an industrial point of view, the improvements achieved through the use of ionic liquids-based ATPS contrasted with traditional ATPS can have a significant impact on the design of a bioprocess. Having a large ATPS pose the challenge of phase components removal, as they become contaminants once the product of interest has been partitioned. A process based on a large ATPS will have the main component of its cost of goods on the unit operation utilized for the removal of the components, as it has been demonstrated previously $[26,27]$. On the other hand, having a small ATPS, as the ionic liquidsbased ATPS (ATPS 4) in this study, implies that the cost of phase components removal will decrease, therefore possibly obtaining an economically attractive industrial process. By decreasing unit operations through the use of ATPS, bioprocesses become less sensitive to changes on the downstream processing yield [27] and can focus on the improvement of the titer in the fermentation stage.

\section{Conclusion}

This research has demonstrated the capabilities of bioprocess modeling and economic analysis to compare and contrast novel and traditional recovery ATPS-based techniques. This work studied the use of a range of aqueous two phase systems (ATPS), including a novel system based on the use of ionic liquids, to recover tetracycline from the fermentation broth of Streptomyces aureofaciens. The ionic liquid based method proved to be the best alternative in terms of DSP yield and $\mathrm{CoG} / \mathrm{g}$, and also provided a biocompatible alternative since ILs obtained using bio-precursors were used. PEG is a well-established GRAS biomolecule, but for Tetracycline recovery, $[\mathrm{Ch}] \mathrm{Cl}$ provided a better economic alternative, moreover, subsequent purification for TC is still needed and the ionic liquid will be removed.

This study focused on already established ATPS in batch mode and using published data for stainless steel containers, because of this, additional research is needed to evaluate the impact of single-use technology for this pharmaceutical product. It is a growing trend in the biotechnological sector as it decreases costs for cleaning and labor, while making more efficient the flow between unit operations.

This study serves to provide an impetus for research on the wider use of IL-based ATPS to improve recovery, whilst lowering the ecological impact of the production of high value-added products.

\section{Acknowledgements}

The authors report no conflicts of interest. Mario A. Torres-Acosta acknowledges the support from CONACYT for the fellowship No. 485025 which enabled him to perform experiments during a research stay in the Department of Biochemical Engineering, University College London. Also, the technical support of Andrew Sinclair from Biopharm Services is gratefully acknowledged. This work was developed within the scope of the project CICECO-Aveiro Institute of Materials, POCI-010145-FEDER-007679 (FCT Ref. UID/CTM/50011/2013), financed by national funds through the FCT/MEC and when appropriate co-financed by FEDER under the PT2020 Partnership Agreement. The research leading to reported results has received funding from the European Research Council under the European Union's Seventh Framework Programme (FP7/2007-2013)/ERC grant agreement no. 337753. Jorge F. B. Pereira acknowledges financial support from FAPESP (São Paulo Research Foundation Brazil) through the project 2014/16424-7.

\section{Appendix A. Supplementary data}

Supplementary data associated with this article can be found, in the online version, at https://doi.org/10.1016/j.seppur.2018.04.041.

\section{References}

[1] B. Hamad, The antibiotics market, Nat. Rev. Drug. Discov. 9 (2010) 675-676.

[2] A. Coates, G. Halls, Y. Hu, Novel classes of antibiotics or more of the same? Brit. J. Pharmacol. 163 (2011) 184-194.

[3] M.A. Darken, H. Berenson, R.J. Shirk, N.O. Sjolander, Production of tetracycline by Streptomyces aureofaciens in synthetic media, Appl. Microbiol. 8 (1960) 46-51.

[4] A.E. Asagbra, A.I. Sanni, O.B. Oyewole, Solid-state fermentation production of tetracycline by Streptomyces strains using some agricultural wastes as substrate, World J. Microb. Biot. 21 (2005) 107-114.

[5] M.L. Nelson, S.B. Levy, The history of tetracyclines, Ann. NY Acad Sci. 1241 (2011) $17-32$.

[6] World Health Organization. WHO Model lists of essential medicines. World Health Organization. Available at: http://www.who.int/medicines/publications/ essentialmedicines/en/. 2015. Accessed May 30, 2016.

[7] Weidenheimer JF, River P, Ritter L. Purification of neutral tetracycline. U.S. Patent 2,820,824, 1958.

[8] Kardys JA. Tetracycline purification process. U.S. Patent 3,013,074, 1961.

[9] R.W. Fedeniuk, P.J. Shand, Theory and methodology of antibiotic extraction from biomatrices, J. Chromatogr A. 812 (1988) 3-15.

[10] Y.K. Yau, C.W. Ooi, E.P. Ng, J.C.W. Lan, T.C. Ling, P.L. Show, Current applications of aqueous two-phase systems, Bioresources Bioprocess. 2 (2015) 49-61.

[11] A.L. Grilo, M.R. Aires-Barros, A.M. Azevedo, Partitioning in aqueous two-phase systes: fundamental, applications and trends, Sep. Purif. Rev. 45 (2016) 68-80.

[12] V. Paquet, M. Myint, C. Roque, P. Soucalle, Partitioning of pristinamycins in aqueous two-phase systems: a first setp toward the development of antibiotic production by extractive fermentation, Biotecnol. Bioeng. 44 (1994) 445-451.

[13] Y. Wang, J. Han, X. Xu, S. Hu, Y. Yan, Partition behavior and partition mechanism of antibiotics in etanol/2-propanol-ammonium sulfate aqueous two-phase systems, Separat. Purificat. Technol. 75 (2010) 352-357.

[14] J.F.B. Pereira, F. Vicente, V.C. Santos-Ebinuma, J. Araújo, A. Pessoa, M.G. Freire, J.A.P. Coutinho, Extraction of tetracycline from the fermentation broth using aqueous two-phase systems composed of polyethylene glycol and cholinium-based salts, Process Biochem. 48 (2013) 716-722.

[15] R.D. Rogers, C.B. Bauer, Partitioning behavior of group 1 and 2 cations in poly (ethylene glycol)-based aqueous biphasic systems, J. Chromatogr. B Biomed. Sci. Appl. 680 (1996) 237-241.

[16] A.M. Azevedo, P.A.J. Rosa, I.F. Ferreira, M.R. Aires-Barros, Chromatography-free recovery of biopharmaceuticals through aqueous two-phase processing, Trends Biotechnol. 27 (2009) 240-247.

[17] H.D. Willauer, J.G. Huddleston, R.D. Rogers, Solute partitioning in aqueous biphasic systems composed of polyethylene glycol and salt: the partitioning of small neutral organic species, Ind. Eng. Chem. Res. 41 (2002) 1892-1904.

[18] J.A. Asenjo, B.A. Andrews, Aqueous two-phase systems for protein separation: a perspective, J. Chromatograph. A. 1218 (2011) 8826-8835.

[19] C.C. Ibarra-Herrera, M.A. Torres-Acosta, G.I. Mendoza-Ochoa, J.M. Aguilar-Yañez, M. Rito-Palomares, Recovery of major royal jelly protein 1 expressed in Pichia pastoris in aqueous two-phase systems, J Chem Technol Biotechnol. 89 (2014) 941-947.

[20] M. González-González, R.C. Wilson, M. Rito-Palomares, Elimination of contaminants from cell preparations using aqueous two-phase partitioning, Sep. Purif. Technol. 158 (2016) 103-107.

[21] M. González-González, M. Rito-Palomares, O. Mendez, Partition behavior of CD133 + stem cells from human umbilical cord blood in aqueous two-phase systems: In route to establish a novel stem cell primary recovery strategies, Biotechnol Progr. 30 (2014) 700-707.

[22] R.R.G. Soares, A.M. Azevedo, J.M. van Alstine, M.R. Aires-Barros, Partitioning in aqueous two-phase systems: analysis of strengths, weakness, opportunities and threats, Biotechnol. J. 10 (2015) 1158-1169.

[23] M.G. Freire, A.F.M. Cláudio, J.M.M. Araújo, J.A.P. Coutinho, I.M. Marrucho, 
J.N. Canongia Lopes, L.P.N. Rebelo, Aqueous biphasic systems: a boost brought about by using ionic liquids, Chem. Soc. Rev. 41 (2012) 4966-4995.

[24] S. Dreyer, U. Kragl, Ionic liquids for aqueous two-phase extraction and stabilization of enzymes, Biotechnol. Bioeng. 99 (2008) 1416-1424.

[25] P.A.J. Rosa, A.M. Azevedo, S. Sommerfeld, W. Backer, M.R. Aires-Barros, Aqueous two-phase extraction as a platform in the biomanufacturing industry: Economical and environmental sustainability, Biotechnol. Adv. 29 (2011) 559-567.

[26] M.A. Torres-Acosta, J.M. Aguilar-Yañez, M. Rito-Palomares, N.J. Titchener-Hooker, Economic analysis of royalactin production under uncertainty: evaluating the effect of parameter optimization, Biotechnol. Prog. 31 (2015) 744-749.

[27] M.A. Torres-Acosta, J.M. Aguilar-Yáñez, M. Rito-Palomares, N.J. Titchener-Hooker, Economic analysis of uriciase production under uncertainty: Contrast of chromatographic purification and aqueous two-phase extraction, Biotechnol. Prog. 32 (2015) 126-133.

[29] S. Shahriari, L.C. Tomé, J.M.M. Araújo, L.P.N. Rebelo, J.A.P. Coutinho, I.M. Marrucho, M.G. Freire, Aqueous biphasic systems: a benign route using cholinium-based ionic liquids, RSC Adv. 3 (2013) 1835-1843.

[30] S.P.M. Ventura, C.S. Marques, A.A. Rosatella, C.A.M. Afonso, F. Gonçalves, J.A.P. Coutinho, Toxicity assessment of various ionic liquid families toward Vibrio fischeri marine bacteria, Ecotox Environ. Safe. 76 (2012) 162-168.

[31] S.P.M. Ventura, A.M.M. Gonçalves, T. Sintra, J.L. Pereira, F. Gonçalves, J.A.P. Coutinho, Designing ionic liquids: the chemical structure role in the toxicity, Ecotoxicology 22 (2013) 1-12.

[32] S.P.M. Ventura, F.A. Silva, A.M.M. Gonçalves, J.L. Pereira, F. Gonçalves, J.A.P. Coutinho, Ecotoxicity analysis of cholinium-based ionic liquids to Vibrio fischeri marine bacteria, Ecotox Environ. Safe. 102 (2014) 48-54.

[33] S.S. Farid, J. Washbrook, N.J. Titchener-Hooker, Decision-support tool for assessing biomanufacturing strategies under uncertainty: Stainless steel versus disposable equipment for clinical trial material preparation, Biotechnol. Prog. 21 (2005) 486-497.

[34] A.C. Lim, Y. Zhou, J. Washbrook, A. Sinclair, B. Fish, R. Francis, N.J. TitchenerHooker, S.S. Farid, Application of a decision-support tool to assess pooling strategies in perfusion culture processes under uncertainty, Biotechnol. Prog. 21 (2005) 1231-1242.

[35] A.C. Lim, J. Washbrook, N.J. Titchener-Hooker, S.S. Farid, A computer-aided approach to compare the production economics of fed-batch and perfusion culture under uncertainty, Biotechnol. Bioeng. 93 (2006) 687-697.

[36] S. Liu, A.S. Simaria, S.S. Farid, L.G. Papageorgiou, Designing cost-effective biopharmaceutical facilities using mixed-integer optimization, Biotechnol. Prog. 29 (2013) 1472-1483.

[37] S. Liu, A.S. Simaria, S.S. Farid, L.G. Papageorgiou, Mathematical programming approaches for downstream processing optimization of biopharmaceuticals, Chem. Eng. Res. Des. 94 (2015) 18-31.

[38] T. Shanklin, K. Roger, P.K. Yegneswaran, M.R. Marten, Selection of bioprocess simulation software for industrial applications, Biotechnol. Bioeng. 72 (2001) 483-489.

[39] E. Heinzle, A.P. Biwer, C.L. Cooney, Development of Sustainable Bioprocesses: Modeling and Assessment (1st edition), John Wiley \& Sons Inc, England, 2006.

[40] Y. Yang, S.S. Farid, N.F. Thornhill, Data mining for rapid prediction of facility fit and debottlenecking of biomanufacturing facilities, J. Biotechnol. 179 (2014) 17-25.

[41] S.S. Farid, Process economics of industrial monoclonal antibody manufacture, J. Chromatogr B. 848 (2007) 8-18.

[42] E. George, N.J. Titchener-Hooker, S.S. Farid, A multi-criteria decision-making framework for the selection of strategies for acquiring biopharmaceutical manufacturing capacity, Comput. Chem. Eng. 31 (2007) 889-901.

[43] J. Pollock, S.V. Ho, S.S. Farid, Fed-batch and perfusion culture processes: Economic, environmental, and operational feasibility under uncertainty, Biotechnol. Bioeng. 30 (2012) 1-14.

[44] J.F.B. Pereira, L.P.N. Rebelo, R.D. Rogers, J.A.P. Coutinho, M.G. Freire, Combining ionic liquids and polyethylene glycols to boost the hydrophobic-hydrophilic range of aqueous biphasic systems, Phys. Chem. Chem. Phys. 15 (2013) 19580-19583.

[45] C.P. Song, R.N. Ramanan, R. Vijayaraghavan, D.R. MacFarlane, E.S. Chan, C.W. Ooi, Green, aqueous two-phase systems based on cholinium aminoate ionic liquids with tunable hydrophobicity and charge density, ACS Sustain. Chem. Eng. 3 (2015) 3291-3298.

[46] Y. Chen, Y. Meng, J. Tang, T. Sun, F. Sun, S. Liang, Extraction of uricase from Candida utilis by applying polyethylene glycol $(\mathrm{PEG}) /\left(\mathrm{NH}_{4}\right) \mathrm{SO}_{4}$ aqueous two-phase system, Afr. J. Biotechnol. 9 (2010) 4788-4795. 